Background GI intolerance is one of the major causes of failure of NSAID treatment for arthritis patients. There has been a large number of studies to examine the risk of NSAID-induced GI side effects in US and Europe, but few studies in Japan.

Objectives In order to evaluate the GI tolerability of NSAIDs in OA patients in Japan, a systematic review of Japanese randomised controlled trials was performed.

Methods This meta-analysis consisted of double blind randomised controlled trials with 4-weeks NSAID treatment in OA patients in Japan. The analysis was composed of 25 trials with 26 types of NSAIDs, among which 6 types were used in more than two trials. The endpoints evaluated in the meta-analysis included incidence of any side effects, any digestive side effects and symptoms indicative of upper GI symptoms such as abdominal pain, nausea and dyspepsia. The general variance-based method was used to estimate the risk of these endpoints. Additionally, to evaluate the effect of risk factors for GI discomfort, odds ratios (ORs) of side effects by sex and age were estimated for individual treatment arms and then combined.

Results The analysis included a total of 4,725 patients. On average, cumulative incidences of patients who had experienced any side effects and any digestive side effects were 14.3\% (95\% CI: 13.3-15.3\%) and 10.4\% (95\% CI: 9.4-11.4\%), respectively. Incidence of any side effects by type of NSAID were as follows: diclofenac $75 \mathrm{mg} /$ day, 19.9\% (10 trials), indomethacin $75 \mathrm{mg} /$ day, $16.2 \%$ (12 trials), acemetacin $90 \mathrm{mg} /$ day, $12.6 \%$ (2 trials), nabumetone $800 \mathrm{mg} /$ day, $9.2 \%$ (2 trials), fenbufen $600 \mathrm{mg} /$ day $8.8 \%$ ( 2 trials), and ibuprofen $900 \mathrm{mg} / \mathrm{day}, 7.8 \%$ (2 trials). Incidence of any digestive side effects were as follows: diclofenac, $16.6 \%$, indomethacin, $10.2 \%$, nabumetone, $8.3 \%$, fenbfen, $6.9 \%$, and ibuprofen $900 \mathrm{mg} /$ day, 6.4\%. Abdominal pain, nausea/vomiting and dyspepsia were the three most common digestive side effects. The cumulative incidence for the upper GI symptoms was estimated to be approximately 10.9\%. Comparing the risk of upper GI symptoms between male and female, the summary OR was 1.71 (95\% CI: 1.11- 2.65). Females were at significantly higher risk than males in the incidence of upper GI symptoms. Comparing the risk of upper GI symptoms between patients under 59 years and above 60 years old, the summary OR was 1.07 (95\% CI: 0.75-1.52).

Conclusion Despite the incidence of side effects varying across the different types of NSAIDs being used, there was an obvious increased risk of GI symptoms. Abdominal pain, nausea/vomiting and dyspepsia were the three most common GI discomforts and this trend is similar to the evidence in US and Europe. Females show a higher risk of side effects and are approximately 50\% more likely to have side effects compared to males. Older patients tend to have a higher risk of side effects, but this is not statistically significant.

\section{SAT0066 STUDYING OF THEW EFFICIENCY OF THE SYNVISK IN OSTEOARTROSIS}

LG Groppa, M Moshneaga. Department of Internal Medicine, State University of Medicine and Pharmacy, Chisinau, The Republic of Moldova

10.1136/annrheumdis-2001.441

Background At present for improvement of life quality in patients with osteoartrosis is necessary to solve some problems: precluding of development of degenerative process in cartilage of affected joint, relieving of pain and improvement of the function of the joint.

\section{Objectives}

Methods For these aims we studied the efficiency of the chondroprotective drug Synvisk by Boenhringer Ingelheim in 25 patients with arthrosis of the knee in medium age of $61,2 \pm 1,7$ years, 16 women and 8 men, with duration of the disease $5,8 \pm$ 0,9 years.

Results Conform X-ray data the distribution of the patients by stages of the disease was as follow: I grade ? 20\%, II grade ? $64 \%$, and III grade ? $16 \%$. In experimental group the drug was administered intraarticular by blind method in dose of $2,0 \mathrm{ml}$ one time in a week, 3 injections for the course, which were repeated after 6 and 12 months. The control group was matched by gender, duration of disease and X-ray date; in this group was administrated placebo conforming same scheme.

Evaluation of the effectiveness and tolerability of compared drugs was carried out in evolution of clinical (pain, functional index by Lesquesne, volume of movement on affected joints), Xray parameters and functional methods (ultrasonography, scintigraphy with Te99m.

In the basic group were mentioned decreasing of the pain intensity and improvement of the joint functions after single injection in one third of the patients, while in control groups similar cases were absent. After 3 courses of the treatment in the basic group the effects were obtained in $86,7 \%$ of the patients, including $33,3 \%$ very good effect, in $53,4 \%$ moderate effect and only in $13,3 \%$ the effects were absent. In control group there were no effect in $80 \%$ of the patients, and in $20 \%$ of the patients there were mentioned moderate effects; good effects were are not observed.

Conclusion Thus, a presented result suggests that administration of Synvisk lead to significant improvement of the life quality in the patients with arthrosis of the knee.

\section{SAT0067 SERUM CARTILAGE OLIGOMERIC MATRIX PROTEIN (COMP) AS A PROGNOSTIC MARKER IN KNEE OSTEOARTHRITIS}

V Vilim, M Olejarova, S Machacek, J Gatterova, K Pavelka. Department Research, Institute of Rheumatology, Praha, Czech Republic

\subsection{6/annrheumdis-2001.442}

Background Three previously published studies aimed to relate rediographic disease progression in OA to baseline serum COMP levels (1-3). In two studies, the authors found no correlation between baseline serum COMP levels and subsequent progression of knee osteoarthritis $(\mathrm{OA}) .^{1,2}$ In the study of hip OA, however, the authors found significant correlation between baseline serum COMP levels and yearly mean narrowing of joint space width. ${ }^{3}$

Objectives To evaluate serum COMP level measured at baseline with a new sandwich ELISA as a predictor of disease progression of knee OA.

Methods Patients with established OA $(n=54)$ represented placebo-taking group from finished and now opened 3 -years long double-blind drug trial. Standing anthero-posterior radiographs of both knees were taken at the beginning and at the end of the study and used to measure joint space width at the medial compartment. COMP was quantified by newly developed sandwich ELISA with monoclonal antibodies 16-F12 and 17-C10.

Results The increased serum COMP level at baseline correlated with 3-years change of joint space width (expressed as the sum 